\title{
Acute pancreatitis-like bile leakage around the portal vein system after endoscopic ultrasound-guided choledochoduodenostomy
}
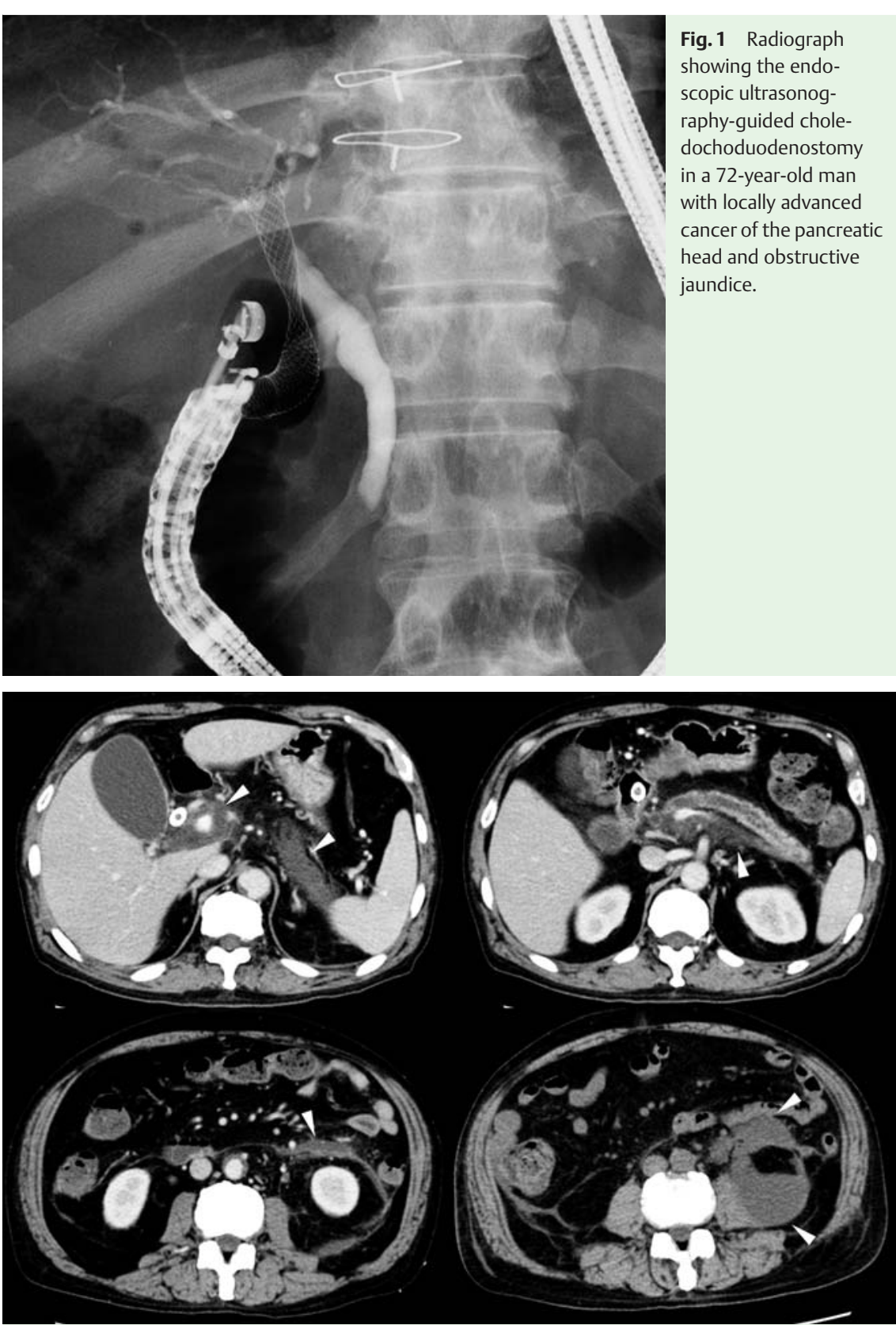

Fig.2 Radiographs showing an acute pancreatitis-like low-density area around the portal vein system and extending to the perirenal space (arrowheads).

The complication rate of endoscopic ultrasound-guided choledochoduodenostomy (EUS-CDS) has been reported to be from $13.6 \%$ to $16 \%[1,2]$. We present a rare case of acute pancreatitis-like bile leakage around the portal vein system after EUSCDS for malignant obstruction of the distal bile duct.
A 72-year-old man with locally advanced cancer of the pancreatic head and obstructive jaundice was referred to our hospital. We successfully performed EUSCDS with a partially covered self-expandable metallic stent (SEMS) (WallFlex, 10× $60 \mathrm{~mm}$; Boston Scientific Japan, Tokyo, Japan); there were no perioperative com-

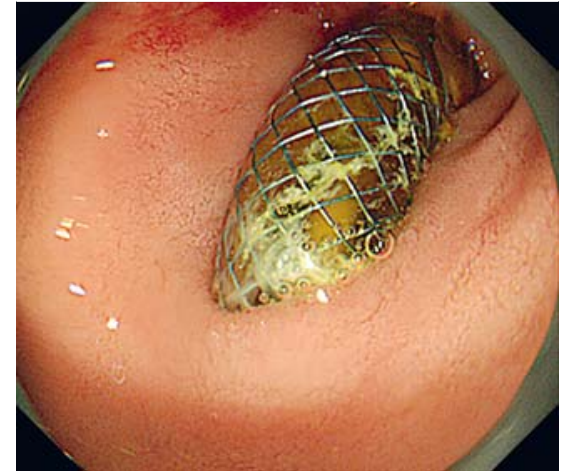

Fig. 3 Endoscopic image showing no dislocation of the self-expandable metallic stent at the duodenal bulb.

plications ( $\bullet$ Fig. 1, $\bullet$ Video 1). However, 7 days later, a high fever developed.

Abdominal computed tomography revealed an acute pancreatitis-like area of low density around the portal vein system that extended to the perirenal space (৫ Fig.2). Esophagogastroduodenoscopy showed no dislocation of the SEMS ( $\bullet$ Fig.3). Laboratory data revealed no elevation of the pancreatic enzymes; the amylase level was $8 \mathrm{U} / \mathrm{L}$ (normal range $50-159)$ and the lipase level was $37 \mathrm{U} / \mathrm{L}$ (normal range $13-49 \mathrm{U} / \mathrm{L}$ ), suggesting that the bile leakage was occurring along the SEMS. The fever resolved in 7 days with antibiotic treatment. However, on day 25 after the procedure, the high fever suddenly recurred.

Abdominal computed tomography showed exacerbation of the abscess at the ventral aspect of the stomach and in the perirenal space. Therefore, we performed EUS-guided drainage with a dedicated, fully covered SEMS (Nagi stent, $1.6 \times 3 \mathrm{~cm}$; Taewoong Medical, Seoul, South Korea). After 5 days, direct endoscopic necrosectomy ( Fig.4a, $\bullet$ Video 1 ) and singletransluminal-gateway transcystic multiple drainages were performed ( $\bullet$ Fig. $4 \mathbf{b}$, - Video 1). The abscess was also drained percutaneously ( $\bullet$ Fig.5). After complete resolution of the abscess, the SEMS and drainage catheters were removed. Afterward, the patient received chemotherapy. Complications of EUS-CDS, such as pneumoperitoneum and bile leakage, have been reported $[1,2]$. The use of a covered 


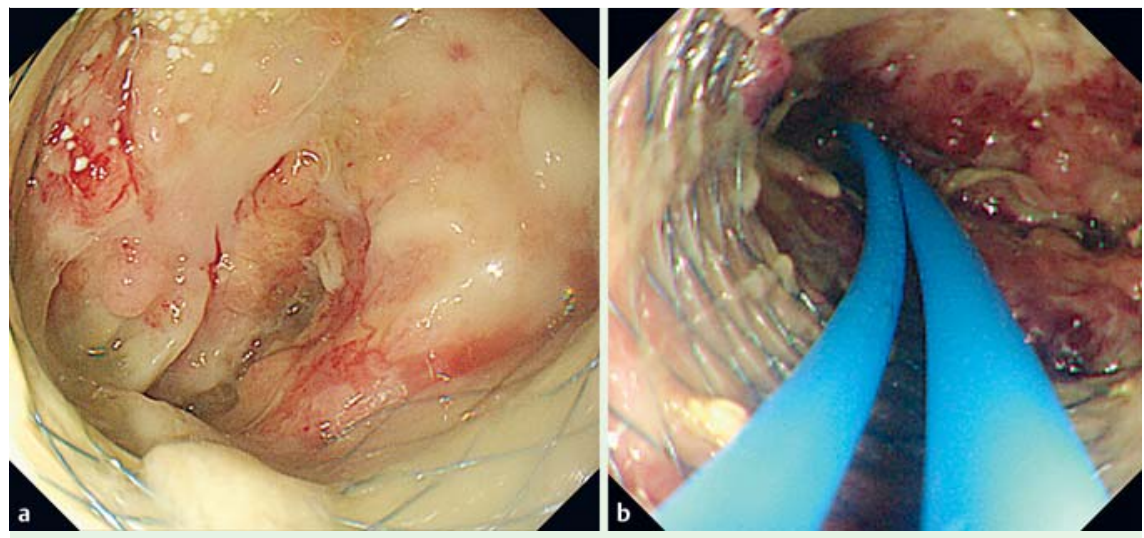

Fig. 4 Endoscopic images. a Necrotic tissue and pus after endoscopic ultrasonography-guided drainage with a dedicated, fully covered self-expandable metallic stent. b Single-transluminal-gateway transcystic multiple drainages.
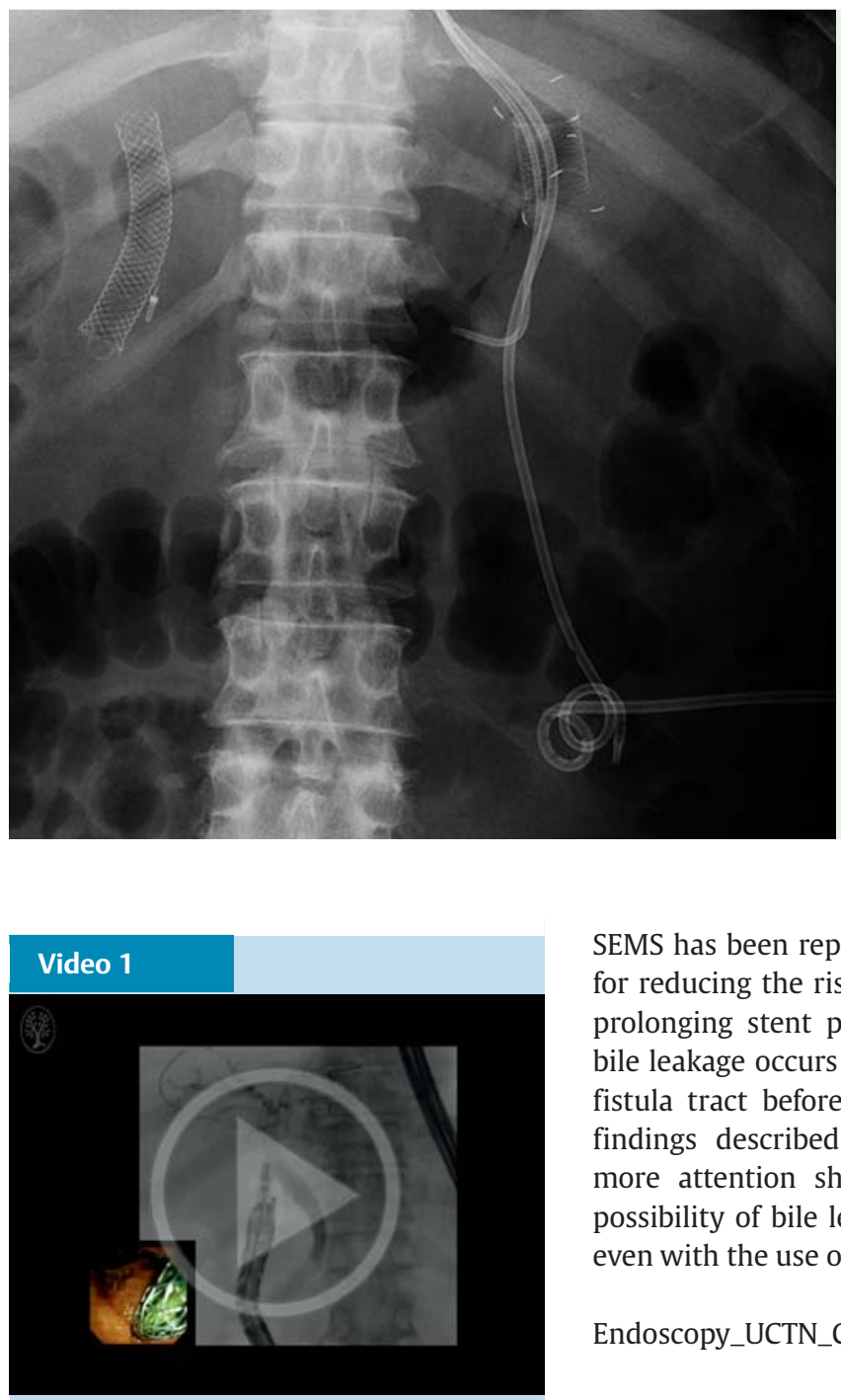

Endoscopic ultrasound-guided choledochoduodenostomy with a partially covered selfexpandable metallic stent followed by direct endoscopic necrosectomy and single-transluminal-gateway transcystic multiple drainage technique through a Nagi stent.

Competing interests: None
Fig. 5 Radiograph showing additional percutaneous catheter placement for abscess drainage.

Hiroshi Kawakami ${ }^{1}$, Masaki Kuwatani ${ }^{2}$, Kazumichi Kawakubo ${ }^{3}$, Yoshimasa Kubota', Shuhei Kawahata', Kimitoshi Kubo', Naoya Sakamoto ${ }^{3}$

${ }^{1}$ Department of Gastroenterology and Hepatology, Hokkaido University Hospital, Sapporo, Japan

${ }^{2}$ Division of Endoscopy, Hokkaido University Hospital, Sapporo, Japan

${ }^{3}$ Department of Gastroenterology and Hepatology, Hokkaido University Graduate School of Medicine, Sapporo, Japan

\section{References}

1 Kawakubo K, Isayama $H$, Kato $H$ et al. Multicenter retrospective study of endoscopic ultrasound-guided biliary drainage for malignant biliary obstruction in Japan. J Hepatobiliary Pancreat Sci 2014; 21: 328 -334

2 Ogura T, Higuchi K. Technical tips of endoscopic ultrasound-guided choledochoduodenostomy. World J Gastroenterol 2015; 21: E820-E828

Bibliography

DOI http://dx.doi.org/

10.1055/s-0034-1392500

Endoscopy 2015; 47: E346-E347

(c) Georg Thieme Verlag KG

Stuttgart · New York

ISSN 0013-726X

\section{Corresponding author}

Hiroshi Kawakami, MD, PhD

Department of Gastroenterology and Hepatology Hokkaido University Hospital

Kita 14

Nishi 5

Kita-ku

Sapporo 060-8648

Japan

Fax: +81-11-706-7867

SEMS has been reported and is preferred for reducing the risk for bile leakage and prolonging stent patency [1]. Generally, bile leakage occurs during dilation of the fistula tract before SEMS insertion. The findings described herein suggest that more attention should be paid to the possibility of bile leakage after EUS-CDS, even with the use of a covered SEMS.

Endoscopy_UCTN_Code_CPL_1AL_2AD 